\title{
Prognostic factors for salvage endoscopic resection for esophageal squamous cell carcinoma after chemoradiotherapy or radiotherapy alone
}

Authors

Institutions
Shinya Kondo ${ }^{1,2}$, Masahiro Tajika², Tsutomu Tanaka², Takeshi Kodaira ${ }^{3}$, Nobumasa Mizuno ${ }^{4}$, Kazuo Hara ${ }^{4}$, Susumu Hijioka ${ }^{4}$, Hiroshi Imaoka ${ }^{4}$, Hidemi Goto ${ }^{5}$, Kenji Yamao ${ }^{4}$, Yasumasa Niwa ${ }^{2}$

Institutions are listed at the end of article. submitted 12. January 2016 accepted after revision 23. May 2016

\section{Bibliography}

Dol http://dx.doi.org/ 10.1055/s-0042-109609 Published online: 9.8.2016 Endoscopy International Open 2016; 04: E841-E848

(c) Georg Thieme Verlag KG Stuttgart . New York E-ISSN 2196-9736

\section{Corresponding author}

Shinya Kondo, MD

Department of

Gastroenterology

Aichi Cancer Center

Aichi Hospital

18 Kuriyado

Kakemachi

Okazaki

Aichi 444-0011

Japan

Fax: +81-564-216467

sy-kondo@acc-aichi.com
License terms

(이요 $\odot \circledast$
Background and study aims: Endoscopic resection is one treatment option for residual or locally recurrent esophageal cancer after definitive chemoradiotherapy or radiotherapy alone. However, little is known about the clinical benefit of salvage endoscopic resection for these lesions. Therefore, the effectiveness and prognostic factors of salvage endoscopic resection were investigated.

Patients and methods: A total of 37 patients with esophageal squamous cell carcinoma (SCC) who underwent salvage endoscopic resection after definitive chemoradiotherapy or radiotherapy alone were reviewed. The method of salvage endoscopic resection was endoscopic mucosal resection using a cap (EMR-C), strip biopsy, or endoscopic submucosal dissection. The effectiveness and prognostic factors of salvage endoscopic resection were retrospectively analyzed.

Results: A total of 37 patients with 49 lesions underwent salvage endoscopic resection. Baseline clinical stages were I in 23 patients, II in 3 pa-

\section{Introduction \\ $\nabla$}

Recently, definitive chemoradiotherapy has become one of the treatment options for esophageal squamous cell carcinoma (SCC) [1-3]. Definitive radiotherapy alone has also been one of the treatment options for mucosal esophageal SCC $[4,5]$. However, local recurrence after chemoradiotherapy or radiotherapy remains a major problem. Some reports have shown the effectiveness of salvage esophagectomy after definitive chemoradiotherapy as additional treatment [6-12]. However, salvage esophagectomy is reported to have higher mortality and complication rates than radical esophagectomy with or without neoadjuvant therapy $[9,10,13]$.

Endoscopic treatment is a minimally invasive procedure. Recently, endoscopic resection has been considered to be one of the curative options for residual or recurrent esophageal SCC after tients, III in 9 patients, and IV in 2 patients. The number of locoregional recurrences and residual lesions were 35 and 14, respectively. The curative en bloc resection rate was $53.1 \%$ (26/49). The total incidence of complications was $18.9 \%$ (7/37); all were successfully managed conservatively. The 3-year and 5-year overall survival rates were $72.9 \%$ and $53.3 \%$, respectively, with a median follow-up period of 54 months. Baseline clinical T1-2 and N0 were significant factors for good prognosis in terms of overall survival on univariate analysis.

Conclusions: Salvage endoscopic resection, especially EMR-C, is a safe and feasible procedure to control residual or recurrent superficial esophageal SCC after definitive chemoradiotherapy or radiotherapy alone. The present results showed that baseline clinical T1-2 and N0 before chemoradiotherapy or radiotherapy were significant prognostic factors.

definitive chemoradiotherapy and radiotherapy when it is localized to the superficial layer. However, little is known about the usefulness, indications, and prognostic factors of endoscopic resection for residual or recurrent tumor after chemoradiotherapy or radiotherapy [14-18]. The aim of this study was to investigate the effectiveness and prognosis of salvage endoscopic resection with a larger number of patients than previous reports.

\section{Patients and methods \\ $\nabla$}

Patients

A database of all patients with esophageal SCC at the Aichi Cancer Center Hospital, Aichi, Japan from January 2000 to May 2010 was retrospectively analyzed. A total of 544 patients with esophageal SCC received definitive chemoradiotherapy or radiotherapy. 
The definitive chemoradiotherapy or radiotherapy consisted of at least 50-Gy irradiation, regardless of concurrent chemotherapy. Most chemotherapeutic regimens comprised two cycles of continuous infusion of 5-fluorouracil and cisplatin or nedaplatin with concurrent radiation (data not shown). A patient with renal dysfunction was treated with low-dose docetaxel. Locoregional recurrence was defined as a cancer relapse at the primary site, and metachronous SCC was defined as a cancer relapse at a site away from the primary site more than 6 months after the initial treatment.

\section{Staging and follow-up}

Pretreatment staging of the esophageal cancers was determined using the tumor-node-metastasis (TNM) classification of the International Union Against Cancer, 7th edition (2009). Staging involved endoscopy with iodine staining, esophagography, and contrast-enhanced neck-to-abdomen computed tomography (CT). Lymph node metastasis was defined as more than $10 \mathrm{~mm}$ in diameter on CT. Complete response was defined as no tumor at follow-up endoscopy with biopsy and neck-to-abdomen CT 3 to 6 weeks after completion of initial treatment. After the confirmation of complete response, follow-up endoscopy with iodine staining was scheduled every 3 months for the first year, every 4 months for the next year, and every 6 months thereafter. Neckto-abdomen CT was performed to detect lymph node or distant metastases every 3 months for the first year, every 6 months for the next 2 years, and annually thereafter.

Complete follow-up information until death or September 2014 was available for all patients.

The effectiveness of salvage endoscopic resection was retrospectively analyzed. Written, informed consent was obtained from all patients. This study was approved by the Institutional Review Board at Aichi Cancer Center Hospital (2014-1-095) and was carried out in accordance with the Declaration of Helsinki.

\section{Endoscopic resection}

Salvage endoscopic resection was defined as endoscopic resection for a recurrent or residual lesion at the primary site after definitive chemoradiotherapy or radiotherapy. It was based on the methods of endoscopic mucosal resection using a cap (EMR-C), strip biopsy, or endoscopic submucosal dissection (ESD). The indication for salvage endoscopic resection was histologically proven SCC by biopsy, endoscopically diagnosed depth of epithelium to two-thirds layer of the submucosa, and the lesion involving less than two-thirds of the esophageal circumference. Lesions that showed the shape of a submucosal tumor or were ulcerative were excluded. They were diagnosed as invading the muscularis propria or more. White light imaging and iodine staining were performed for diagnosis of locoregional and residual lesions. Endoscopic ultrasound (EUS) had not been performed as a staging modality for these lesions because of diagnostic difficulty. There were no distant and/or lymph node metastases on CT. All endoscopic resection treatments were performed with the patients under intravenous sedation with midazolam (Astellas Pharma Co., Tokyo, Japan) and pethidine (Mitsubishi Tanabe Pharma Co., Osaka, Japan).

\section{Endoscopic mucosal resection (EMR)}

EMR was performed by the EMR-C method and the strip biopsy method [19]. For EMR-C, a forward-viewing endoscope (GIFQ240 or GIF-Q260J; Olympus Medical Systems, Tokyo, Japan) with a plastic cap (MH-594 or MH-595; Olympus) on its tip was introduced. Saline solution was injected into the submucosa beneath the lesion with an injection needle. A crescent-moonshaped snare (SD-221L-25; Olympus) was opened within the plastic cap, and the lesion was aspirated into the cap. The snare was then closed, and a forced coagulation current was applied to resect the lesion ( $\mathbf{F i g . 1}$ ). For the strip biopsy method, a doublechannel endoscope (GIF-2T240; Olympus) was required. After saline solution injection into the submucosa, a snare and grasping forceps were each inserted through a channel. The forceps were then passed through the opened snare, and the snare was closed lightly around them. An area near the lesion was grasped with the forceps to evaluate the lesion, the snare was opened, the lesion was strangulated, and the tumor was then resected by applying an electrosurgical current. After resection, iodine staining was performed to check for a residual lesion. If a residual lesion was found, additional piecemeal resection or argon plasma coagulation (APC) (ICC-200; Erbe Elektromedizin Ltd, Tübingen, Germany) was performed.

\section{Endoscopic submucosal dissection (ESD)}

ESD was started from August 2008 for residual or recurrent esophageal SCC after chemoradiotherapy [20]. The indication for ESD was a lesion more than $10 \mathrm{~mm}$ in diameter on endoscopy. ESD was performed using a Flush-Knife $^{\circledR}$ (DK2618JN20, DK2618JN10; Fujifilm Co., Ltd., Tokyo, Japan) or a ball tip bipolar needle-knife (B-Knife ${ }^{\circledR}$ ) (BSJB15B, Zeon Medical Co., Ltd., Tokyo, Japan). The choice of the specific modality was decided by the endoscopist who performed the procedure. A forward-viewing endoscope (GIF-Q260J; Olympus) with a transparent attachment (D-201-11804; Olympus) on its tip was introduced.

\section{Histologic evaluation and assessment of therapeutic efficacy}

Resected specimens cut into 2-mm-thick slices were examined histologically according to the guidelines for the diagnosis and treatment of esophageal cancer by the Japan Esophageal Society [21]. R0 resection was defined as en bloc resection with tumorfree horizontal margins. $\mathrm{R} 1$ resection was defined as a positive resection margin for tumor cells. Curative resection was defined as a tumor that was resected en bloc with a tumor-invasion depth of epithelium to submucosa, with no lymphovascular involvement and tumor-free margins.

\section{Complications}

Bleeding related to the procedure was defined as bleeding that required postoperative endoscopic hemostasis, such as endoscopic clipping or thermocoagulation. Perforation was diagnosed as mediastinal connective tissue that was observed during the procedure. A postoperative stricture was defined as a stricture that required endoscopic balloon dilation because of symptoms.

\section{Statistical analysis}

Overall survival was calculated from the date of first salvage endoscopic resection to the occurrence of death or to the date of last follow-up. Overall survival included deaths from any cause. For statistical analysis, Mann-Whitney's $U$ test was used. Actual survival was calculated by the Kaplan-Meier method. A Cox proportional hazards regression model was used for univariate analysis of the simultaneous effects of prognostic factors. Therefore, the measure of association in this study was the hazard ratio along with the $95 \%$ confidence interval (95\%CI). In all analyses, a $P$ value $<0.05$ was accepted as significant. All statistical analyses 


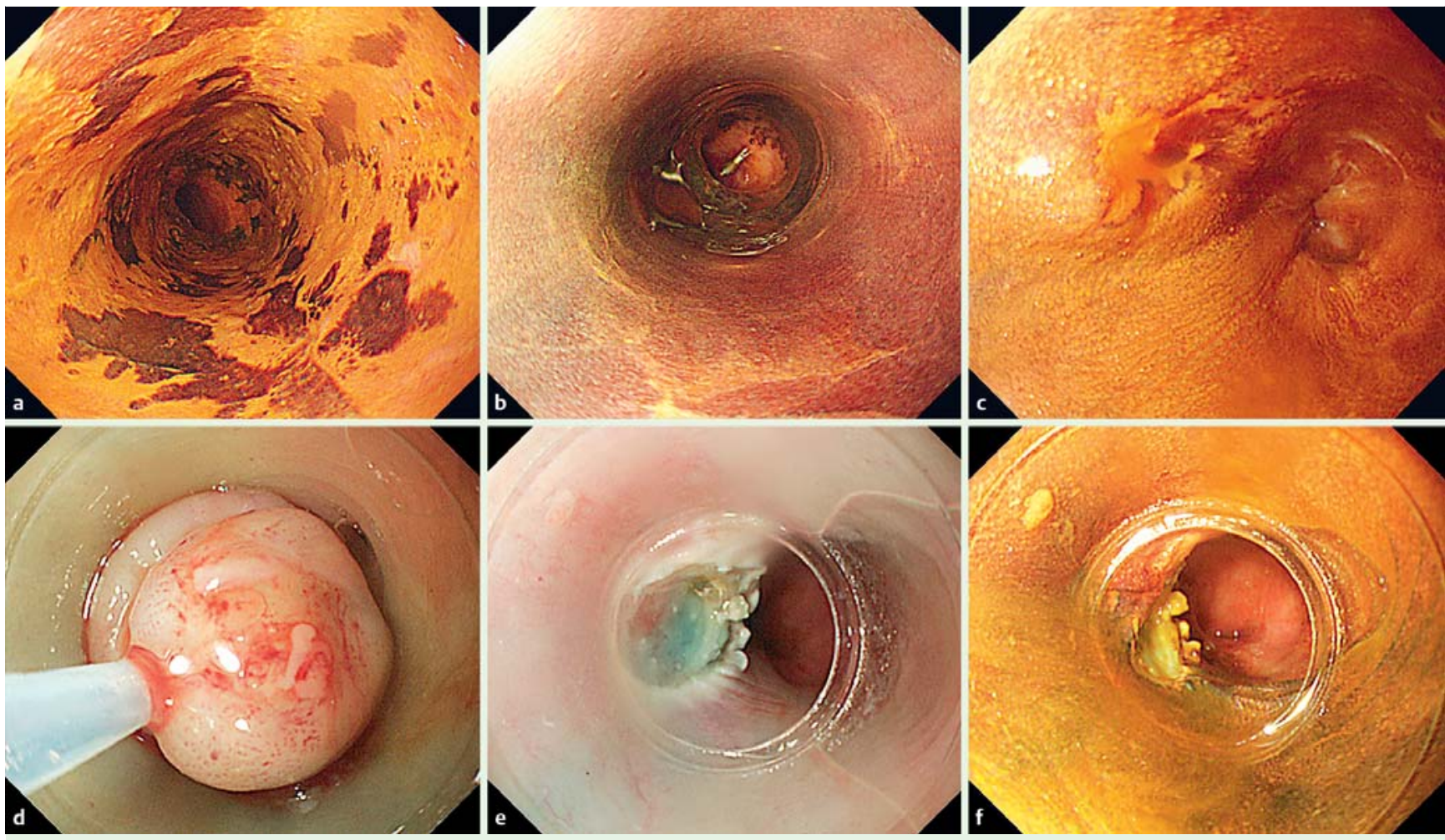

Fig. 1 Salvage endoscopic mucosal resection using a cap (EMR-C) for locoregional recurrence after chemoradiotherapy. a Endoscopy after iodine staining shows a circumferential, slightly depressed lesion in the lower thoracic esophagus. b Complete response is achieved after chemoradiotherapy. $\mathbf{c}$ Twenty-four months after chemoradiotherapy, locoregional recurrence occurs on the same site. $\mathbf{d}$ The lesion aspirated into the cap is strangulated by a snare. $\mathbf{e}$ The lesion is resected. $\mathbf{f}$ lodaine staining is performed to check for a residual lesion.

were performed using the SPSS statistical software package 11.0 (SPSS Inc., Chicago, Illinois, United States).

\section{Results \\ $\nabla$}

\section{Patients}

Of the 544 patients, 294 achieved a complete response, and 82 of the 294 patients thereafter developed locoregional or metachronous SCC without lymph node or distant metastatic recurrence until March 2011 ( Fig.2). Of the 82 patients with local recurrence, 52 had locoregional recurrence, and 30 had metachronous SCC. Patients with endoscopically diagnosed superficial type, for which endoscopic resection could be indicated, included 37 with locoregional recurrence. Of these 37 patients, 27 underwent salvage endoscopic resection. On the other hand, 250 of 544 patients did not achieve a complete response, of whom 11 patients remained with a localized residual primary lesion in the shape of the superficial type without metastatic findings ( $\bullet$ Fig.2); 10 of them underwent salvage endoscopic resection. Three patients with local superficial recurrence and one patient with superficial residual tumor underwent APC because of their underlying disease (severe cardiac disease and severe cirrhosis among others). Thirty-seven patients (36 males, one female) underwent salvage endoscopic resection because of locoregional recurrence or residual disease ( Fig.2). Pretreatment patient characteristics are shown in Table 1 . Their median age was 66 years (range 50-84 years). The middle thoracic area was the most frequent primary site. The number of patients with clinical stages I, II, III, and IV was 23, 3, 9, and 2, respectively. The details of chemoradiotherapy and radiotherapy are shown in $\bullet$ Table2. Twenty- eight patients were post-chemoradiotherapy, while nine were post-radiotherapy alone. All patients who underwent radiotherapy alone were clinical T1. Complete response was achieved in $71 \%(20 / 28)$ in the chemoradiotherapy group and $77 \%(7 / 9)$ in the radiotherapy group. The median time from the first day of radiation therapy to confirming local relapse by endoscopy for these 27 patients who achieved a complete response was 11.3 months (range 4.4-74.9 months).

\section{Lesions}

The lesions' characteristics for salvage endoscopic resection are summarized in Table3. Six patients were found to have two lesions, and two patients were found to have four lesions concurrently. Thus, 37 patients with 49 lesions underwent salvage endoscopic resection. The lower thoracic area was the most frequent region. The majority of lesions were 0-IIc type and mucosal depth on endoscopy, with 35 locoregional recurrences and 14 residual lesions.

\section{Results of endoscopic resection}

The endoscopic resection results are shown in 0 Table 4. Among the 49 lesions, the EMR-C method was performed for 44 cases, the strip biopsy method was performed for two, and the remaining three cases underwent ESD. The selection depended on the skill of the investigator and the period. Before February 2008, all salvage endoscopic resection procedures were performed by the EMR method. After that, the ESD method was used for large lesions. Forty lesions (81.6\%) were histologically confirmed to be mucosal lesions, seven lesions (14.3\%) showed submucosal invasion, and two lesions were unknown due to the burning effect. 


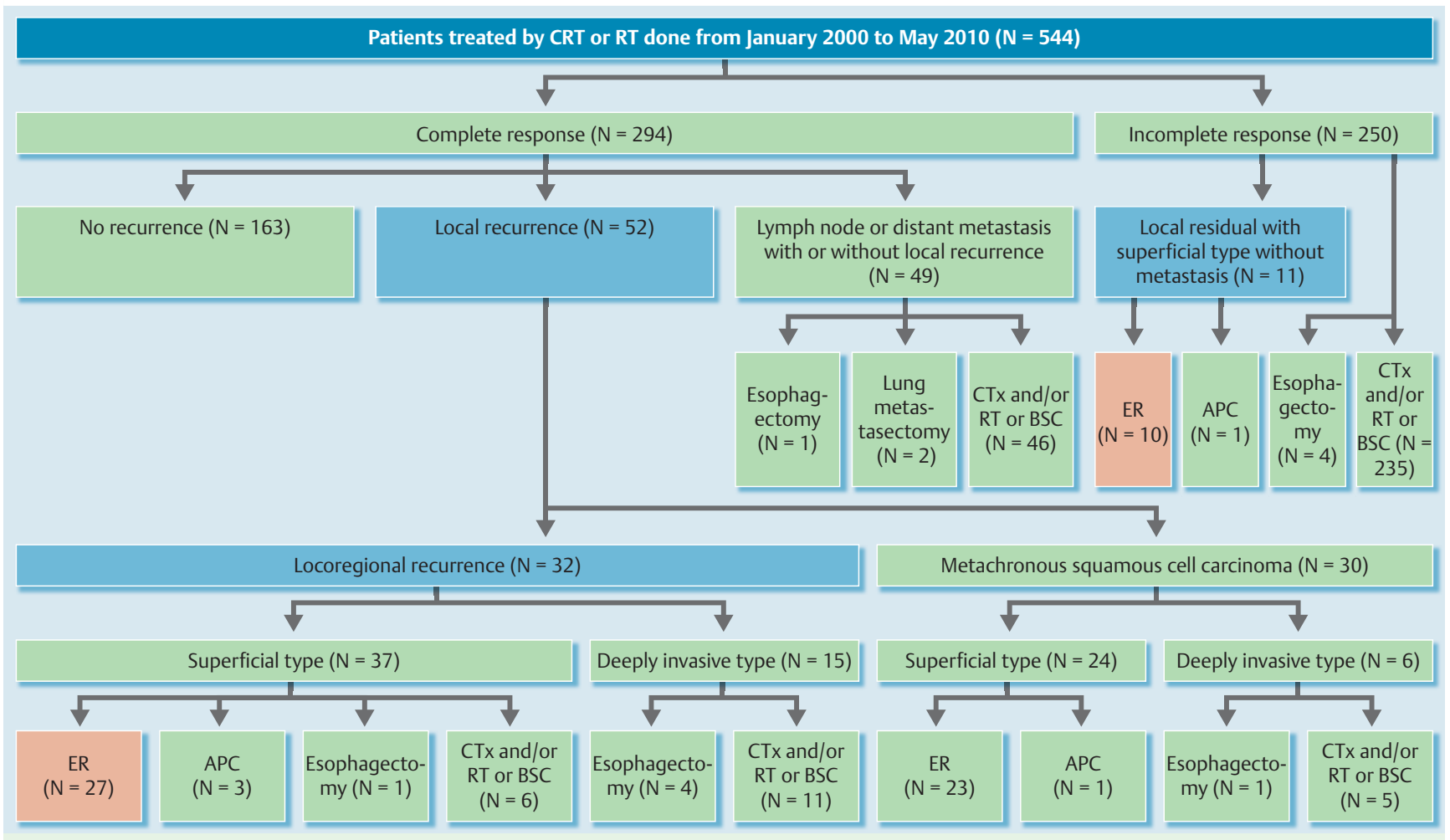

Fig. 2 Representative flow chart of the patient sample assessed in this study. CRT, chemoradiotherapy; ER, endoscopic resection; RT, radiotherapy; APC, argon plasma coagulation; CTx, chemotherapy; BSC, best supportive care.

Curative resection was obtained for 26 lesions (53.1\%). The curative resection rate with ESD was $100 \%(3 / 3)$.

\section{Complications of endoscopic resection}

The overall complication rate was $18.9 \%$ (7/37). Four patients developed postoperative strictures, all of whom were successfully treated by endoscopic balloon dilation. One case of postoperative bleeding occurred and was successfully treated by endoscopic coagulation therapy. One perforation with mediastinal emphysema occurred. The patient recovered well, with no oral ingestion for 5 days and antibiotic administration for 7 days. One case of aspiration pneumonia occurred and was successfully treated by intravenous administration of antibiotics. After EMR-C/strip biopsy/ESD, postoperative bleeding occurred in $1 / 0 / 0$, perforation occurred in $1 / 0 / 0$, aspiration pneumonia occurred in $0 / 0 / 1$, and postoperative stricture occurred in 3/0/1, respectively. There were no treatment-related deaths with salvage endoscopic resection.

\section{Follow-up data and survival}

The median follow-up period of all 37 patients was 54 months (range, 3.2-116.1 months). All patients were followed-up for at least 3 years or until death.

The clinical course of all lesions after salvage endoscopic resection is summarized in Fig. 3. During the follow-up period, 15 patients had no recurrence. However, eight of them died of other diseases; five of the eight died of head and neck cancer. Local recurrence was observed in 14 patients after salvage endoscopic resection, of whom 11 patients were successfully treated by additional endoscopic resection. Two patients underwent esophagectomy as an additional treatment. Lymph node and/or distant metastatic recurrences were found in eight patients after salvage endoscopic resection. The prognosis after metastatic recurrence was dismal. Most patients died within 1 year. During the followup period, 16 patients were alive, all of whom were disease-free, and 21 patients died; 11 patients died from progression of esophageal cancer, and the others died from head and neck cancer, liver cirrhosis, and other causes.

The 3-year and 5-year overall survival rates were $72.9 \%$ and $53.3 \%$, respectively ( Fig. 4 ).

Univariate analysis was performed to identify independent predictors of overall survival for all patients. On both analyses, baseline clinical stage T1 - 2 and N0 were significant factors for a good prognosis compared with T3-4 and N1 - 3 ( Table 5). Recurrent or residual tumor, tumor location, and curative resection or not with salvage endoscopic resection were also not significant in terms of overall survival.

\section{Discussion \\ $\nabla$}

In the present study, two important things about endoscopic resection were demonstrated in esophageal SCC after definitive chemoradiotherapy or radiotherapy alone. First, salvage endoscopic resection, especially EMR-C, was a safe and minimally invasive procedure to control locally recurrent or residual lesions and to preserve the esophagus itself. Second, baseline clinical stages T1-2 and N0 were significantly associated with a good prognosis.

First, salvage endoscopic resection, especially EMR-C, was safe and helpful to control local recurrent or residual lesions. The overall complication rate was $18.9 \%$ (7/37), which not low, but four of the seven cases were stenoses. The occurrence of complications might be influenced not only by endoscopic resection, 
Table 1 Patients' baseline characteristics before chemoradiotherapy or radiotherapy $(n=37)$.

\begin{tabular}{|c|c|}
\hline Median age (range), years & $66(50-84)$ \\
\hline \multicolumn{2}{|l|}{ Sex } \\
\hline Male & 36 \\
\hline Female & 1 \\
\hline \multicolumn{2}{|l|}{ HNC } \\
\hline Negative & 25 \\
\hline Positive & 12 \\
\hline \multicolumn{2}{|l|}{ Location } \\
\hline Cervical & 1 \\
\hline Upper thoracic & 3 \\
\hline Middle thoracic & 17 \\
\hline Lower thoracic & 12 \\
\hline Upper-middle thoracic & 1 \\
\hline Middle-lower thoracic & 1 \\
\hline Cervical-lower thoracic & 1 \\
\hline Upper thoracic + lower thoracic & 1 \\
\hline \multicolumn{2}{|l|}{ Primary tumor } \\
\hline cT1 & 28 \\
\hline cT2 & 1 \\
\hline cT3 & 3 \\
\hline cT4 & 5 \\
\hline \multicolumn{2}{|l|}{ Regional lymph nodes } \\
\hline cNO & 24 \\
\hline cN1 & 9 \\
\hline cN2 & 4 \\
\hline cN3 & 0 \\
\hline \multicolumn{2}{|l|}{ Distant metastasis } \\
\hline cM0 & 35 \\
\hline cM1 & 2 \\
\hline \multicolumn{2}{|l|}{ Clinical stage } \\
\hline cStage I & 23 \\
\hline cStage II & 3 \\
\hline cStage III & 9 \\
\hline cStage IV & 2 \\
\hline
\end{tabular}

HNC, previous or recently discovered concurrent head and neck cancers.

but also by initial radiotherapy or chemoradiotherapy. The adverse event rate of salvage EMR was almost equivalent to that of ordinary EMR (mainly using EMR-C) without chemoradiotherapy or radiotherapy [22]. Postoperative stricture, postoperative bleeding, and perforation occurred in $8.8 \%(3 / 34), 2.9 \%(1 / 34)$, and $2.9 \%(1 / 34)$ of cases in this salvage EMR group excluding three ESD patients and 7.8\% (14/179), $1.7 \%(3 / 179)$, and $1.1 \%$ (2/179) of cases in the ordinary EMR group, respectively. The safety, overall survival, and cause-specific survival of this study could be regarded as acceptable.

Second, the present study also showed that baseline clinical stage $\mathrm{T} 1-2$ and NO were significantly associated with a good prognosis on univariate analysis. During the follow-up period, 16 patients were alive ( $\bullet$ Fig. 3 ). The survival rates for T1 -2 and T3 -4 were $55.2 \%(16 / 29)$ and $0 \%(0 / 8)$, respectively. The survival rates for N0 and $\mathrm{N} 1-3$ were $100 \%(16 / 16)$ and $0 \%(0 / 21)$, respectively. From this result, initial T1-2 and N0 appear to be a good indication for salvage endoscopic resection. There has been a report that the prognosis of baseline clinical stage T1-2 was better than that of T3-4 before salvage photodynamic therapy [23]. However, no report has analyzed the prognostic factors of salvage endoscopic resection. To the best of our knowledge, this is the first study to report them. On the other hand, salvage endoscopic resection for baseline clinical T3-4 patients was completed mainly using EMR-C. The curative resection rate for these lesions
Table 2 Initial treatment before salvage endoscopic resection $(n=37)$.

\begin{tabular}{|c|c|c|}
\hline & $\begin{array}{l}\text { Chemoradiotherapy } \\
(n=28)\end{array}$ & $\begin{array}{l}\text { Radiotherapy } \\
(\mathrm{n}=9)\end{array}$ \\
\hline \multicolumn{3}{|l|}{ Radiotherapy (Gy) } \\
\hline Median & 60 & 60 \\
\hline Range & $50.4-64$ & $60-66$ \\
\hline \multicolumn{3}{|l|}{ Chemotherapy } \\
\hline 5FU + cisplatin & 20 & \\
\hline 5FU + nedaplatin & 5 & \\
\hline Cisplatin & 1 & \\
\hline $5 F U$ & 1 & \\
\hline Docetaxel & 1 & \\
\hline Complete response & 20 & 7 \\
\hline Partial response & 8 & 2 \\
\hline
\end{tabular}

5FU, 5-fluorouracil.

Table 3 Tumor characteristics of salvage endoscopic resection (49 lesions in 37 patients).

\begin{tabular}{|c|c|}
\hline \multicolumn{2}{|l|}{ Tumor status } \\
\hline Residual & 14 \\
\hline Recurrent & 35 \\
\hline \multicolumn{2}{|l|}{ Tumor location } \\
\hline Cervical & 2 \\
\hline Upper thoracic & 4 \\
\hline Middle thoracic & 20 \\
\hline Lower thoracic & 23 \\
\hline \multicolumn{2}{|l|}{ Macroscopic type } \\
\hline 0 -IIc & 45 \\
\hline 0 -Is & 4 \\
\hline \multicolumn{2}{|c|}{ Depth with endoscopic findings } \\
\hline Mucosal & 39 \\
\hline Submucosal & 10 \\
\hline
\end{tabular}

0-Ilc, slightly depressed type; 0-Is, sessile (broad-based) type.

was $37.5 \%$ (3/8). Aspirating the lesion into the cap might be effective for these fibrotic lesions. All T3 -4 patients died after salvage endoscopic resection during the follow-up period. However, the median survival rate was 24.7 months (range, 3.2 - 69.3 months). Furthermore, $37.5 \%$ (3/8) of T3 -4 patients survived more than 3 years after salvage endoscopic resection. These results show that salvage endoscopic resection might not be appropriate for T3-4 patients, although it seems meaningful for these patients.

The present results indicated that whether curative resection was achieved with salvage endoscopic resection was not related to overall survival. After salvage endoscopic resection, there were 14 cases of local re-recurrence, and 11 of these 14 patients underwent additional salvage endoscopic resection; of the three other patients, two of them underwent esophagectomy when diagnosed as clinical $\mathrm{T} 2$, and the other one was given best supportive care because of underlying renal disease. Six of 11 lesions were locoregional recurrent or residual lesions after salvage endoscopic resection. Pathological findings at re-salvage endoscopic resection indicated that one was a curative resection, but five were not. Although it may be difficult to completely resect locally recurrent or residual lesions by endoscopic resection, performing additional salvage endoscopic resection might improve these patients' overall survival. Furthermore, most of these patients' conditions were not perfect because of disease progression and adverse events of chemoradiotherapy. For this reason, 
Table 4 Clinical results of salvage endoscopic resection (49 lesions in 37 patients).

\begin{tabular}{|l|l|}
\hline Method of endoscopic resection & 44 \\
\hline EMR-C & 2 \\
\hline Strip biopsy & 3 \\
\hline ESD & \\
\hline Resection type & 40 \\
\hline En bloc resection & 9 \\
\hline Piecemeal resection & \\
\hline Adverse events & 1 \\
\hline Postoperative bleeding & 1 \\
\hline Perforation & 1 \\
\hline Pneumonia & 4 \\
\hline Stricture & \\
\hline Histological evaluation & 29 \\
\hline R0 resection & 11 \\
\hline R1 resection & 9 \\
\hline Unknown (piecemeal, burned) & \\
\hline Curative resection rate & 26 \\
\hline Curative resection & 23 \\
\hline Non-curative resection & $11(3-35)$ \\
\hline Median tumor size (range), mm & 37 \\
\hline Depth of histological invasion & 3 \\
\hline EP-LPM & 2 \\
\hline MM & 5 \\
\hline SM1 & 2 \\
\hline SM2 or more & \\
\hline Unknown (burned) & \\
\hline
\end{tabular}

EMR-C, endoscopic mucosal resection using a cap; ESD, endoscopic submucosal dissection; R0, tumor-free margins with en bloc resection; R1, tumor-positive margins with en bloc resection; EP, epithelium; LPM, lamina propria mucosae; MM, muscularis mucosae; SM, submucosal layer.

salvage endoscopic resection and additional salvage endoscopic resection seem to make a great deal of sense to decrease tumor volume, maintain good condition, preserve the esophagus, and improve the outcome. We might consider it as palliative treatment.

The present study showed that salvage endoscopic resection was a safe procedure compared with some reports of salvage surgery [6-12]. However, we should pay attention to the indications for salvage endoscopic resection. It is important to know that salvage endoscopic resection is not indicated for all locoregional or residual lesions. It is known that not all recurrent and residual lesions originate from the mucosa. In some cases, cancer cells are

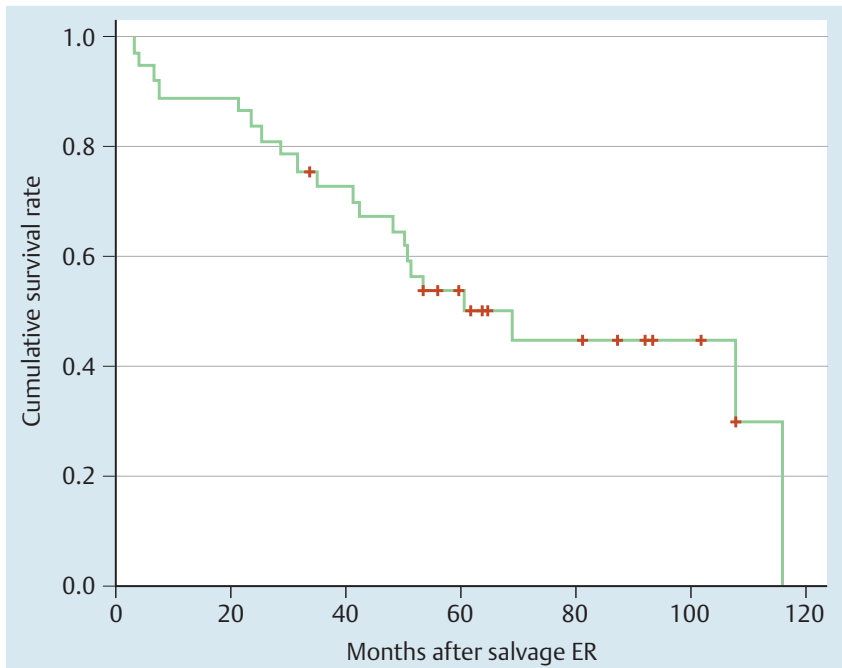

Fig. 4 Overall survival curve for the 37 patients who underwent salvage endoscopic resection.

left in the deep layer of the esophageal wall after radiotherapy. Especially when the baseline clinical stage is T2 or more, recurrent or residual lesions might exist mainly in the submucosal or deeper layer. It has been reported that these lesions showed the shape of a submucosal tumor in many cases [24]. Furthermore, most esophageal intramural metastases are also submucosal in shape. Therefore, in cases of lesions like a submucosal tumor, we have to consider photodynamic therapy or surgery as a salvage treatment procedure. Salvage photodynamic therapy has the possibility to cure deeper lesions than salvage endoscopic resection, and it is less invasive than salvage surgery $[23,25,26]$. However, it has the drawbacks of high rates of severe stenosis, perforation, and phototoxicity, and pathological evaluation is not possible. Salvage surgery can also cure deeper lesions than salvage endoscopic resection and with pathological evaluation, especially in cases of R0 resection (no residual tumor) [6-12]. However, it has the drawbacks of high adverse events, mortality, and morbidity rates. We believe that choosing the appropriate salvage treatment leads to a good prognosis for these patients. In surveillance of patients after chemoradiotherapy, we also have to pay attention to changes of the esophagus. The layer structures of the esophageal wall are destroyed after chemoradiotherapy.

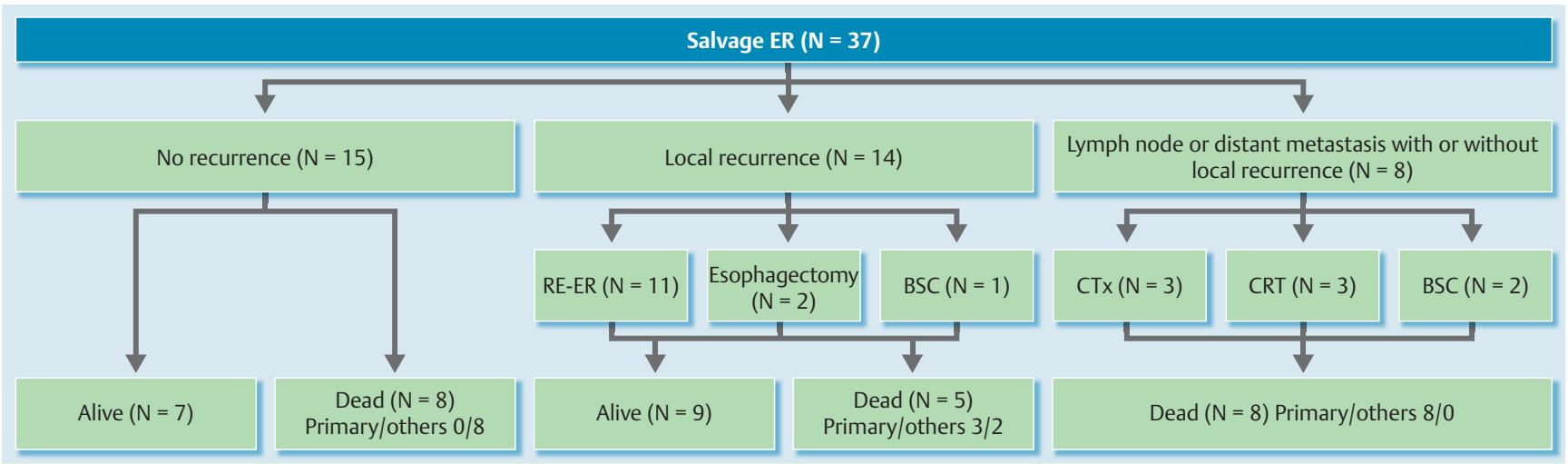

Fig. 3 Clinical course of patients after salvage endoscopic resection $(n=37)$. Primary, died of primary esophageal cancer; others, died of other disease. CRT, chemoradiotherapy; ER, endoscopic resection; CTx, chemotherapy; BSC, best supportive care. 


\begin{tabular}{|c|c|c|c|c|}
\hline Characteristics & Patients (n) & HR & $95 \% \mathrm{Cl}$ & $P$ value \\
\hline Age, years & & 0.766 & $0.272-2.155$ & 0.614 \\
\hline$<70$ & 28 & & & \\
\hline$\geq 70$ & 9 & & & \\
\hline Sex & & 21.651 & $0.001-508836.525$ & 0.549 \\
\hline Male & 36 & & & \\
\hline Female & 1 & & & \\
\hline PS & & 0.441 & $0.169-1.154$ & 0.095 \\
\hline 0 & 26 & & & \\
\hline $1 / 2$ & 11 & & & \\
\hline HNC & & 1.341 & $0.509-3.535$ & 0.553 \\
\hline Negative & 25 & & & \\
\hline Positive & 12 & & & \\
\hline Baseline cT stage & & 0.182 & $0.072-0.458$ & $<0.001$ \\
\hline $\mathrm{cT} 1 / 2$ & 29 & & & \\
\hline cT3/4 & 8 & & & \\
\hline Baseline $\mathrm{cN}$ stage & & 0.151 & $0.057-0.396$ & $<0.001$ \\
\hline cNO & 24 & & & \\
\hline $\mathrm{cN} 1-3$ & 13 & & & \\
\hline Baseline cM stage & & 0.688 & $0.091-5.203$ & 0.717 \\
\hline cM0 & 35 & & & \\
\hline cM1 & 2 & & & \\
\hline Recurrent or residual & & 0.928 & $0.336-2.562$ & 0.885 \\
\hline Recurrent & 27 & & & \\
\hline Residual & 10 & & & \\
\hline Resection pattern & & 1.025 & $0.423-2.484$ & 0.956 \\
\hline Curative & 20 & & & \\
\hline Non-curative & 17 & & & \\
\hline
\end{tabular}

Table 5 Univariate analysis of long-term survival after salvage endoscopic resection $(n=37)$.

HR, hazard ratio; Cl, confidence interval; PS, performance status; HNC, previous or recently discovered concurrent head and neck cancers.

For this reason, endoscopic ultrasound may not be useful to evaluate tumor depth [27 -29]. There may also be difficulty with narrow-band imaging in evaluating lesions for radiation-induced mucosal damage. We think that it is necessary to evaluate them comprehensively with both endoscopic and CT findings. Close surveillance is also important to detect residual or recurrent lesions in the early stage.

The present study has several limitations. First, this study was retrospective, from a single institution, and the sample size was small. Additionally, there were some biases. One possible bias was the unequal population at the baseline clinical stage. Another possible bias was diagnosis of regional lymph node metastases. Previous reports noted that EUS is more sensitive for the detection of regional lymph node metastases of esophageal cancer than CT [30]. By the addition of EUS, the diagnostic power for staging before chemoradiotherapy or radiotherapy will increase. It can also be useful for restaging to exclude synchronous lymph node metastases when we consider salvage endoscopic resection for residual or recurrent lesions. Recently, the usefulness of positron emission tomography combined with CT (PET/CT) for evaluating lymph node metastases of esophageal cancer has been reported. These reports showed that PET/CT has a significantly higher positive predictive value than CT alone [31,32]. Karashima et al. noted that specificity is also higher than CT alone [31]. These results indicate that both EUS and PET/CT should be performed to evaluate lymph node metastases for staging before chemoradiotherapy or radiotherapy and for determining whether salvage endoscopic resection is indicated. Another possible bias was that the salvage endoscopic resection method was affected by the timing of treatment. For the endoscopic treatment of esophageal cancers in our hospital, EMR-C was mainly performed in an earlier period, whereas ESD was started for large lesions from 2008.
Yamashita et al. reported that, for lesions $11 \mathrm{~mm}$ in diameter or larger, ESD was superior to EMR-C in efficacy, as assessed by achieving en bloc resection with tumor-free margins [33]. The European Society of Gastrointestinal Endoscopy Guideline also recommends EMR for lesions smaller than $10 \mathrm{~mm}$ [34]. The present results also showed that all three cases of salvage ESD were en bloc resections. All of them achieved curative resection histologically, which was superior to EMR $(50 \%, 23 / 46)$. Three reports showed the outcomes of salvage ESD [16-18]. The en bloc resection rate and the curative resection rate were $91.6-100 \%$ and $25-68.4 \%$, respectively. All reports showed that perforation and treatmentrelated death did not occur. These results indicate that ESD will be the main procedure for salvage endoscopic resection for EMR in the future. Therefore, when the procedure was performed should have a minimal impact on the results. In addition, data on effectiveness, safety, and long-term outcome for salvage ESD are still lacking in this study. However, it is difficult to plan a study of additional treatments for this patient group. This population is expected to be small. For this reason, the present outcome is clinically quite important to improve the prognosis of these patients. In conclusion, the results of this study show that salvage endoscopic resection, especially EMR-C, is a safe and effective treatment to control recurrent or residual superficial esophageal SCC after definitive chemoradiotherapy or radiotherapy alone. Salvage endoscopic resection may also provide a survival benefit for certain patients, especially those with baseline clinical stage T1 -2 and N0.

Competing interests: None 
Institutions

${ }^{1}$ Department of Gastroenterology, Aichi Cancer Center Aichi Hospital, Aichi, Japan

2 Department of Endoscopy, Aichi Cancer Center Hospital, Aichi, Japan

${ }^{3}$ Department of Radiation Oncology, Aichi Cancer Center Hospital, Aichi, Japan

${ }^{4}$ Department of Gastroenterology, Aichi Cancer Center Hospital, Aichi, Japan

${ }^{5}$ Department of Gastroenterology, Nagoya University Graduate School of Medicine, Nagoya, Japan

\section{References}

1 Kato K, Muro K, Minashi K et al. Phase II study of chemoradiotherapy with 5-fluorouracil and cisplatin for stage II-III esophageal squamous cell carcinoma: JCOG trial (JCOG 9906). Int J Radiat Oncol Biol Phys 2011; 81: 684-690

2 Kato $\mathrm{H}$, Sato A, Fukuda $\mathrm{H}$ et al. A phase II trial of chemoradiotherapy for stage I esophageal squamous cell carcinoma: Japan Clinical Oncology Group Study (JCOG9708). Jpn J Clin Oncol 2009; 39: 638-643

3 Ishida $K$, Ando $N$, Yamamoto $S$ et al. Phase II study of cisplatin and 5 -fluorouracil with concurrent radiotherapy in advanced squamous cell carcinoma of the esophagus: a Japan Esophageal Oncology Group (JEOG)/Japan Clinical Oncology Group trial (JCOG9516). Jpn J Clin Oncol 2004; 34: 615-619

4 Ishikawa H, Sakurai H, Tamaki Yet al. Radiation therapy alone for stage I (UICC T1NOM0) squamous cell carcinoma of the esophagus: indications for surgery or combined chemoradiotherapy. J Gastroenterol Hepatol 2006; 21: 1290-1296

5 Sai H, Mitsumori M, Araki $N$ et al. Long-term results of definitive radiotherapy for stage I esophageal cancer. Int J Radiat Oncol Biol Phys 2005; 62: $1339-1344$

6 Swisher SG, Wynn P, Putnam JB et al. Salvage esophagectomy for recurrent tumors after definitive chemotherapy and radiotherapy. J Thorac Cardiovasc Surg 2002; 123: 175-183

7 Nakamura $T$, Hayashi $K$, Ota $M$ et al. Salvage esophagectomy after definitive chemotherapy and radiotherapy for advanced esophageal cancer. Am J Surg 2004; 188: 261 - 266

8 Tachimori Y. Role of salvage esophagectomy after definitive chemoradiotherapy. Gen Thorac Cardiovasc Surg 2009; 57: 71 - 78

9 Chao YK, Chan SC, Chang HK et al. Salvage surgery after failed chemoradiotherapy in squamous cell carcinoma of the esophagus. Eur J Surg Oncol 2009; 35: 289-294

10 Miyata H, Yamasaki M, Takiguchi S et al. Salvage esophagectomy after definitive chemoradiotherapy for thoracic esophageal cancer. J Surg Oncol 2009; 100: $442-446$

11 Gardner-Thorpe J, Hardwick RH, Dwerryhouse SJ. Salvage oesophagectomy after local failure of definitive chemoradiotherapy. Br J Surg 2007; 94: 1059-1066

12 Tomimaru Y, Yano M, Takachi K et al. Factors affecting the prognosis of patients with esophageal cancer undergoing salvage surgery after definitive chemoradiotherapy. J Surg Oncol 2006; 93: 422 - 428

13 Saeki H, Morita M, Tsuda Y et al. Multimodal treatment strategy for clinical T3 thoracic esophageal cancer. Ann Surg Oncol 2013; 20: $4267-4273$

14 Hattori S, Muto M, Ohtsu A et al. EMR as salvage treatment for patients with locoregional failure of definitive chemoradiotherapy for esophageal cancer. Gastrointest Endosc 2003; 58: 65-70

15 Yano T, Muto M, Hattori S et al. Long-term results of salvage endoscopic mucosal resection in patients with local failure after definitive chemoradiotherapy for esophageal squamous cell carcinoma. Endoscopy 2008; 40: 717-721

16 Saito Y, Takisawa H, Suzuki $H$ et al. Endoscopic submucosal dissection of recurrent or residual superficial esophageal cancer after chemoradiotherapy. Gastrointest Endosc 2008; 67: 355-359
17 Takeuchi M, Kobayashi M, Hashimoto S et al. Salvage endoscopic submucosal dissection in patients with local failure after chemoradiotherapy for esophageal squamous cell carcinoma. Scand J Gastroenterol 2013; 48: $1095-1101$

18 Koizumi S, Jin M, Matsuhashi T et al. Salvage endoscopic submucosal dissection for the esophagus-localized recurrence of esophageal squamous cell cancer after definitive chemoradiotherapy. Gastrointest Endosc 2014; 79: $348-353$

19 Inoue $H$, Endo $M$, Takeshita $K$ et al. A new simplified technique of endoscopic esophageal mucosal resection using a cap-fitted panendoscope (EMRC). Surg Endosc 1992; 6: 264-265

20 Fujishiro M, Yahagi N, Kakushima $N$ et al. Endoscopic submucosal dissection of esophageal squamous cell neoplasms. Clin Gastroenterol Hepatol 2006; 4: $688-694$

21 Kuwano H, Nishimura Y, Oyama T et al. Guidelines for Diagnosis and Treatment of Carcinoma of the Esophagus April 2012 edited by the Japan Esophageal Society. Esophagus 2015; 12: 1-30

22 Kawai $H$, Niwa $Y$, Tajika $M$ et al. Is endoscopic mucosal resection acceptable for Stage 0 or IA esophageal squamous cell carcinoma? Nagoya Med J 2012; 52: 185 - 197

23 Yano T, Muto M, Minashi K et al. Long-term results of salvage photodynamic therapy for patients with local failure after chemoradiotherapy for esophageal squamous cell carcinoma. Endoscopy 2011; 43: 657 663

$24 \mathrm{Tu} \mathrm{CH}$, Muto M, Horimatsu T et al. Submucosal tumor appearance is a useful endoscopic predictor of early primary-site recurrence after definitive chemoradiotherapy for esophageal squamous cell carcinoma. Dis Esophagus 2011; 24: 274-278

25 Yano T, Muto M, Minashi $K$ et al. Photodynamic therapy as salvage treatment for local failures after definitive chemoradiotherapy for esophageal cancer. Gastrointest Endosc 2005; 62: 31-36

26 Wolfsen HC, Hemminger LL. Salvage photodynamic therapy for persistent esophageal cancer after chemoradiation therapy. Photodiagnosis Photodyn Ther 2006; 3: 11 - 14

27 Griffin JM, Reed CE, Denlinger CE. Utility of restaging endoscopic ultrasound after neoadjuvant therapy for esophageal cancer. Ann Thorac Surg 2012; 93: 1855-1859; discussion 1860

28 Kalha I, Kaw M, Fukami $N$ et al. The accuracy of endoscopic ultrasound for restaging esophageal carcinoma after chemoradiation therapy. Cancer 2004; 101: 940-947

29 Zhang X, Watson DI, Lally $C$ et al. Endoscopic ultrasound for preoperative staging of esophageal carcinoma. Surg Endosc 2005; 19: 1618 1621

30 van Vliet EP, Heijenbrok-Kal MH, Hunink MG et al. Staging investigations for oesophageal cancer: a meta-analysis. Br J Cancer 2008; 98: $547-557$

31 Karashima R, Watanabe M, Imamura Yet al. Advantages of FDG-PET/CT over CT alone in the preoperative assessment of lymph node metastasis in patients with esophageal cancer. Surg Today 2015; 45: 471-477

32 Okada M, Murakami T, Kumano S et al. Integrated FDG-PET/CT compared with intravenous contrast-enhanced CT for evaluation of metastatic regional lymph nodes in patients with resectable early stage esophageal cancer. Ann Nucl Med 2009; 23: 73-80

33 Yamashita T, Zeniya A, Ishii $H$ et al. Endoscopic mucosal resection using a cap-fitted panendoscope and endoscopic submucosal dissection as optimal endoscopic procedures for superficial esophageal carcinoma. Surg Endosc 2011; 25: $2541-2546$

34 Pimentel-Nunes P, Dinis-Ribeiro M, Ponchon T et al. Endoscopic submucosal dissection: European Society of Gastrointestinal Endoscopy (ESGE) Guideline. Endoscopy 2015; 47: 829-854 